Лелюхин В. Е., Колесникова О. В., Шпилев А. М., Давыдов Ю. А.

ЦИФРОВЫЕ ТЕХНОЛОГИИ В УПРАВЛЕНИИ ПРОЕКТИРОВАНИЕМ И ПРОИЗВОДСТВОМ

Лелюхин В. Е., Колесникова О. В., Шпилев А. М., Давыдов Ю. А.

V. E. Lelyukhin, O. V. Kolesnikova, A. M. Shpilev, Yu. A. Davydov

ЦИФРОВЫЕ ТЕХНОЛОГИИ В УПРАВЛЕНИИ ПРОЕКТИРОВАНИЕМ И ПРОИЗВОДСТВОМ

\title{
DIGITAL TECHNOLOGIES IN THE MANAGEMENT OF DESIGN AND PRODUCTION
}

Лелюхин Владимир Егорович - кандидат технических наук, доцент кафедры технологии промышленного производства Дальневосточного федерального университета (Россия, Владивосток); 690091, Приморский край, г. Владивосток, ул. Суханова, 8; тел.: 7 (914) 723-52-57. E-mail: lelv0@mail.ru.

Mr. Vladimir E. Lelyukhin - PhD in Engineering, Assistant Professor, School of Engineering, Far Eastern Federal University (Russia, Vladivostok); 690091, Primorsky territory, Vladivostok, 8 Sukhanov str.; tel.: + 7 (914) 72352-57. E-mail: lelv0@mail.ru.

Колесникова Ольга Валерьевна - кандидат технических наук, доцент кафедры технологии промышленного производства Дальневосточного федерального университета (Россия, Владивосток); 690091, Приморский край, г. Владивосток, ул. Суханова, 8; тел.: + 7 (914) 652-97-80. E-mail: miis@mail.ru.

Ms. Olga V. Kolesnikova - PhD in Engineering, Assistant Professor, School of Engineering, Far Eastern Federal University (Russia, Vladivostok); 690091, Primorsky territory, Vladivostok, 8 Sukhanov str.; tel.: + 7 (914) 65297-80. E-mail: miis@mail.ru.

Шпилев Анатолий Михайлович - доктор технических наук, профессор Комсомольского-на-Амуре государственного университета (Россия, Комсомольск-на-Амуре); 681013, Хабаровский край, г. Комсомольскна-Амуре, пр. Ленина, 27. E-mail: Shpilev@knastu.ru.

Mr. Anatoly M. Shpilev - Dr.hab. of Engineering, Professor, Komsomolsk-on-Amur State University (Russia, Komsomolsk-on-Amur); 681013, Khabarovsk territory, Komsomolsk-on-Amur, 27 Lenin str. E-mail: Shpilev@knastu.ru.

Давыдов Юрий Анатольевич - доктор технических наук, профессор, ректор Дальневосточного государственного университета путей сообщения (Россия, Хабаровск); 680021, Хабаровский край, г. Хабаровск, ул. Серышева, 47; тел.: + 7 (4212) 40-75-16. E-mail: rector@festu.khv.ru.

Mr. Yuriy A. Davydov - Doctor of Engineering, Professor, Rector of Far Eastern State Transport University (Russia, Khabarovsk); 680021, Khabarovsk territory, Khabarovsk, 47 Serysheva str.; tel.: + 7 (4212) 40-75-16. E-mail: rector@festu.khv.ru.

Аннотация. В настоящее время машиностроительные предприятия в процессе конструкторскотехнологической подготовки и при выполнении работ широко используют цифровые технологии для эффективного планирования и управления. Разнородность и различная функциональность информационных систем привели к появлению проблем при создании интегрированных систем управления предприятиями и корпорациями. В представленной статье предлагается подход к решению сложившихся проблем. Авторами разработана концепция построения единого информационного пространства на основе функциональной модели предприятия. Базовыми элементами единого информационного пространства являются конструкторско-технологическая информация об изделиях и информация о текущем состоянии производственных ресурсов предприятия. Оригинальность и эффективность предложенной структуры информационного пространства заключается в том, что оно содержит все необходимые и достаточные для управления сведения, а также взаимосвязи между их элементами. Внедрение и практическая эксплуатация системы, основанной на изложенных принципах, позволили значительно повысить эффективность работы на производственном предприятии, выпускающем технологическое оборудование.

Summary. Currently, shipbuilding and ship repair enterprises in the process of design and technological preparation and in the performance of works widely use digital technologies for effective planning and management. The heterogeneity and different functionality of information systems have led to problems in the creation of integrated management systems for enterprises and corporations. In the article presented, the authors propose an approach to solving the existing problems. The authors developed the concept of building a single information space based 
on the functional model of the enterprise. Design and technological information about products and information about the current state of the company's production resources are the basic elements of a single information space. The originality and effectiveness of the proposed structure of the information space is that it contains all the necessary and sufficient information for management, as well as the interrelations between its elements. At the manufacturing plant, which produces equipment for fish processing on ships, we have implemented an integrated system built on the principles described. At the same time, a significant increase in the efficiency of work in the enterprise. Ключевые слова: цифровые технологии, объединённое информационное пространство, автоматизация проектирования, автоматизация управления, интегрированные системы, машиностроительное производство.

Key words: digital technologies, integrated information space, design automation, automation management, integrated systems, shipbuilding.

УДК 004.94:004.057:004.032.34:65.014.1

Работа выполнена при финансовой поддержке Министерства образования и науки Российской Федерации по государственному контракту № 02.G25.31.0173.

\section{Введение}

Характерной чертой многих современных машиностроительных предприятий является мелкосерийный характер производства [1;8].

Повторяемость выпуска изделий или заказов на этих предприятиях зачастую нерегулярна, а иногда отсутствует полностью. С учётом достаточно большого количества разноплановых технологий, значительного разнообразия поставщиков материалов и комплектующих и необходимости увязки между собой всех процессов предприятия с точки зрения управления этот вид производства можно отнести к сложным системам. При этом одной из основных проблем автоматизации управления ресурсами на единичных и мелкосерийных предприятиях с дискретизацией производственных процессов является сложность и реальная вычислимость алгоритмов при наличии множества разноплановых факторов [2; 3; 9].

Для повышения эффективности машиностроительных предприятий необходимо создание действенных систем управления, учитывающих особенности этой отрасли, опыт отечественных разработок, а также использующих российские и зарубежные прогрессивные разработки.

Современное состояние теории и практики автоматизации управления ресурсами сложных производственных систем характеризуется рядом проблем, связанных с дезинтеграцией организационного взаимодействия подразделений и служб; чрезвычайной сложностью, а зачастую и невозможностью стыковки CAD-, PLM- и ERP-систем; отсутствием формальных методов для автоматизации подготовки технологических процессов (бизнес-процессов); корректного производственного планирования при изменении выпускаемой продукции [4].

\section{Проблемы интеграции цифровых технологий}

Первая проблема заключается в дезинтеграции организационного взаимодействия производственных подразделений и служб предприятия, что вызывает разобщённость локальных целевых функций и, как следствие, несогласованность в управляющих воздействиях внутри подсистем.

В практической деятельности предприятий эта проблема проявляется в том, что в подавляющем большинстве недостаточно чёткая информированность об оперативных целях и задачах в конечном итоге приводит к отклонениям в производственном процессе. Для преодоления этой проблемы необходимо отыскать и сформулировать принципы и правила информационного взаимодействия между элементами системы с оперативным изменением локальных целевых функций таким образом, чтобы это обеспечивало достижение глобальной цели.

Вторая проблема касается разобщённости локальных информационных пространств представления данных в системах автоматизации конструирования (CAD), системах автоматизации проектирования технологических процессов (САПР ТП), системах управления конструкторскотехнологическими образами на протяжении всего их жизненного цикла (PLM), а также в системах 
Лелюхин В. Е., Колесникова О. В., Шпилев А. М., Давыдов Ю. А.

ЦИФРОВЫЕ ТЕХНОЛОГИИ В УПРАВЛЕНИИ ПРОЕКТИРОВАНИЕМ И ПРОИЗВОДСТВОМ

управления ресурсами предприятия (ERP). На современном этапе развития эта проблема порождает сложность, а зачастую и невозможность компоновки сквозной интегрированной системы управления потоками информации. Для решения этой проблемы представляется крайне необходимым иметь единое информационное пространство, обобщающее в систематизированном виде необходимую и достаточную информацию для обеспечения полноценного функционирования перечисленных систем автоматизации.

Третья проблема заключается в формализации синтеза единичных технологических процессов для автоматизации подготовки технологической информации в системе управления, что позволит значительно сократить количество ошибок и повысить оперативность технологической подготовки. Для решения указанной проблемы требуется понимание и выявление принципов и формальных методов синтеза и выбора приемлемых технологических решений. Аналогами отечественных систем автоматизации проектирования технологических процессов (САПР ТП) в различных странах мира являются системы CAPP (Computer-Aided Process Planning), проектирование в которых в основном осуществляется на основе использования уже существующих и описанных технологий.

Четвёртая проблема заключается в корректности и адекватности моделей производственного планирования при выпуске часто сменяемой продукции на предприятиях мелкосерийного и единичного характера с учётом реального ресурсного обеспечения. Предлагаемые в мире многочисленные методы и алгоритмы, учитывая NP-сложность задач планирования, обеспечивают решение в условиях наличия ряда ограничений, что не соответствует реальным производственным условиям.

Многие авторы сходятся во мнении, что основная проблема заключается не столько в NP-сложности процесса формирования графика загрузки оборудования, сколько в адекватности используемых моделей организационным и функциональным структурам предприятий.

\section{Концепция единого информационного пространства управления}

Решение проблемы автоматизации управления проектированием и производством машиностроительных изделий необходимо начинать с исследования предприятия и построения функциональной схемы его деятельности.

В предлагаемом подходе функциональная схема включает функции, выполняемые подразделениями предприятия, потоки (материальный, финансовый, информационный), а также описывает преобразование этих потоков в результате выполнения функций.

Модель производства представляет собой базовую производственную площадку в виде конечного множества рабочих мест $R$, на которых в каждый момент времени выполняется некоторая совокупность дискретных детале-операций в соответствии с заданной программой (производственным планом).

Базовую производственную площадку можно представить в виде конечного множества рабочих мест $R$, элементами которого являются $r_{1}, r_{2}, \ldots, r_{n}$.

Каждый элемент этого множества может быть представлен в виде нескольких проекций в векторном пространстве его свойств. Например, любое рабочее место $r_{i}$ может иметь: собственное обозначение $-r_{i}^{N}$; площадь для его размещения $-r_{i}^{S}$; типоразмер оборудования $-r_{i}^{T} ;$ модель оборудования $-r_{i}^{M}$ и т. д. Таким образом, полную информацию об элементе $r_{i}$ представляет собой объединение всех проекций этого элемента $r_{i}=r_{i}^{N} \cup r_{i}^{S} \cup r_{i}^{T} \cup r_{i}^{M}$.

Тогда множество $R$ представляется объединением конечных множеств, имеющих одинаковую мощность:

$$
\begin{gathered}
R=R^{N} \cup R^{S} \cup R^{T} \cup R^{M}, \text { где } R^{N}:=\left\{r_{1}^{N}, r_{2}^{N}, \ldots, r_{n}^{N}\right\} ; \\
R^{S}:=\left\{r_{1}^{S}, r_{2}^{S}, \ldots, r_{n}^{S}\right\} ;
\end{gathered}
$$




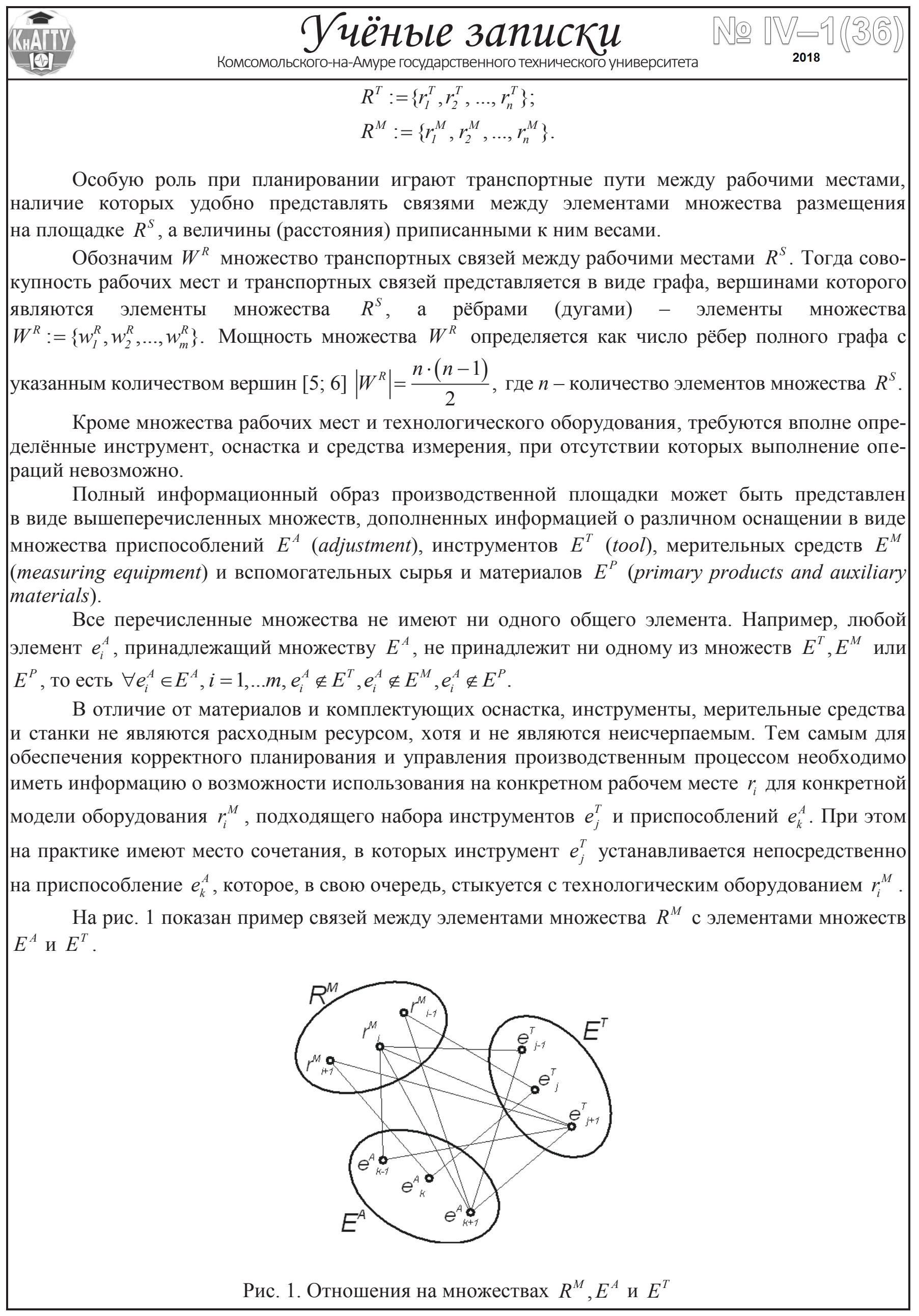


Мощность множества связей $W^{R T}$ между множествами оборудования $R^{M}$ и инструмента $E^{T}$ определится как декартово (прямое) произведение элементов этих непересекающихся множеств:

$$
W^{R T}=R^{M} \times E^{T}=\left\{\left(r, e^{T}\right) \mid r \in R^{M}, e^{T} \in E^{T}\right\} .
$$

Аналогично определяются мощность множества $W^{R A}$ отношений между множествами $R^{M}$ и $E^{A}$, а также множества $W^{A T}$ между $E^{A}$ и $E^{T}$.

Множество $R$ является семейством множеств $R^{j}$. Тогда в соответствии с аксиомой выбора [7] для любого семейства $R$ непустых множеств существует функция которая сопоставляет один из элементов $r_{i}^{J}$ множества $R^{j}$ каждому множеству семейства [10]. Для данного случая функция выбора представляется в следующем виде:

$$
\forall R[\varnothing \notin R \Rightarrow \exists f: R \rightarrow \cup R \forall A \in R(f(A) \in A)] .
$$

Согласно этой аксиоме можно произвольно выбирать из каждого множества один из элементов, совокупность которых, в свою очередь, позволяет сформировать элементы соответствующего семейства.

Таким образом, можно сформировать информационную модель в виде «виртуального отпечатка» производственной площадки, которая является базисом для реализации производственных функций. Несмотря на то что наличие такого базиса является необходимым для существования производства, требуется ещё выполнение условия достаточности $[1 ; 3 ; 4]$.

В данной работе предлагается условие необходимости обеспечить базисом (центром) пространства управления с двумя измерениями $P^{2}$. Одним измерением является перечислимое множество наименований рабочих мест $R^{M}$, другим - время $(t)$.

Для обеспечения достаточности базис пространства управления может быть дополнен любым количеством двухмерных подпространств со связанной с базисом координатой времени, например, пространство инструментов, приспособлений и мерительных средств.

\section{Система автоматизации управления на основе единого информационного пространства}

Единое информационное пространство не определяет какие-либо конкретные решения, но отображает среду существования общего количества различных вариантов решений и позволяет строить модели управления.

Авторы предлагают формировать систему управления на основе ядра управления, построенного в едином информационном пространстве. Ядро управления (core of management) можно представить в виде постоянно изменяющегося во времени набора функций преобразования потоков, размещённых в центре пространства управления $P^{2}$ с двумя измерениями. При этом технологическая операция является элементарной функцией, описывающей связи между значениями параметров на координатных осях и регламентирующей эти значения.

Таким образом, формирование управляющей программы может выполняться в двухмерном пространстве времени-рабочих мест без учёта изменений остальных координат (инструмент, материалы, и т. д.), как показано на рис. 2.

Пространство управления, изображённое на рис. 2, определяется двумя координатами осью рабочих мест $R^{M}$ и осью времени $t$. Прямоугольники отображают размещённые на рабочих местах функции преобразования материальных потоков детале-сборочных единиц во временных интервалах.

При каждой процедуре планирования (добавление или удаление заказов) ядро управления из состояния $C_{k}$ переходит в состояние $C_{k+1}$. 


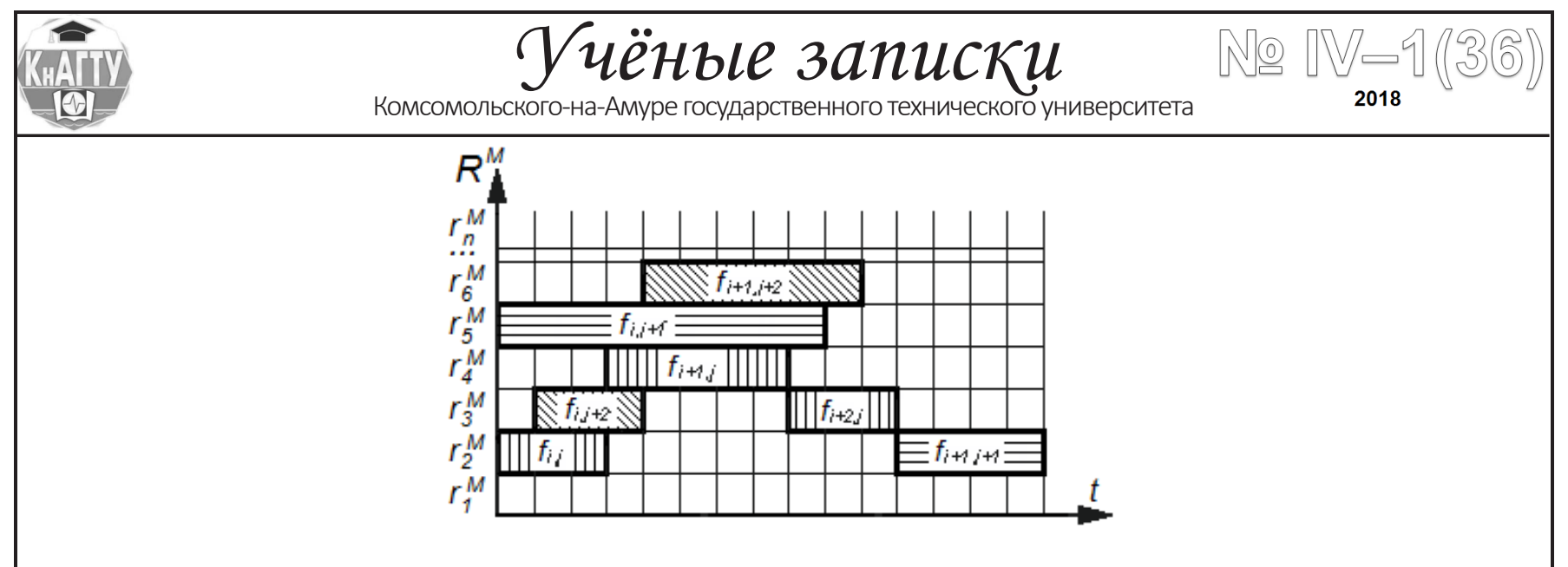

Рис. 2. Отображение ядра управления

в базисе пространства с двумя измерениями $P^{2}$

Далее, пространство управления дополняется двухмерными подпространствами инструментов, приспособлений и мерительных средств со связанной с базисом координатой времени. Тогда проекции функций $f_{i, j}$ на соответствующее подпространство из числа $E^{A}, E^{T}, E^{M}$ или $E^{P}$ формируют потребности в соответствующем оснащении. На рис. 3 показан пример визуализации проекции функций ядра управления на подпространство $E^{T}$.

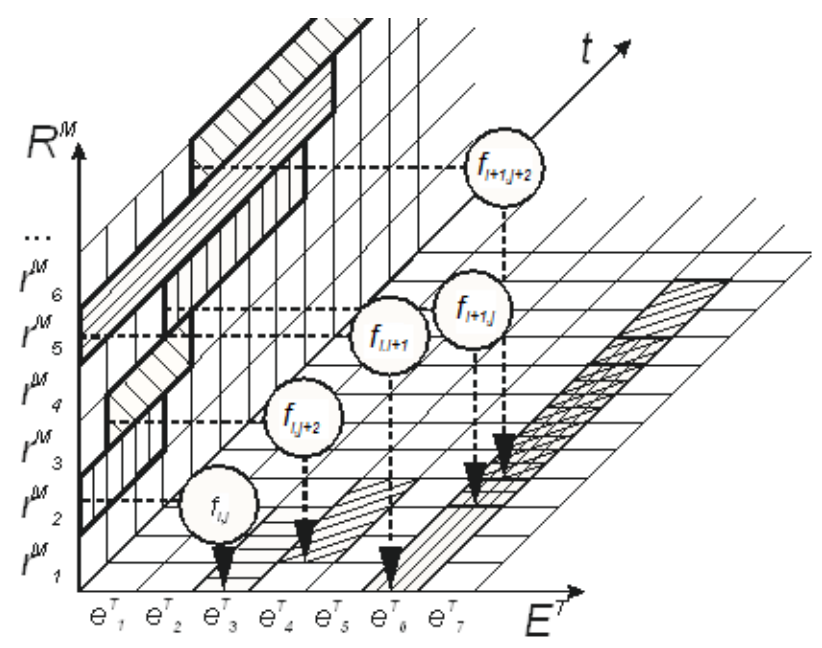

Рис. 3. Проекции функций ядра управления на подпространство $E^{T}$

Такой подход предполагает формирование программы управления в виде графика загрузки рабочих мест при следующих условиях: координата времени бесконечна, координата рабочих мест счётна, конечна и ограничена имеющимся на предприятии оборудованием; координаты инструментов, материалов, оснастки являются счётными, конечными, но считаются неограниченными. В данном случае под неограниченностью понимается то, что на практике потребное количество ресурса $K^{P}$ всегда меньше либо равно предельно допустимому значению $\left[K^{P}\right]$, то есть $K^{P} \leq\left[K^{P}\right]$.

Можно сказать, что ядро управления представляет собой насыщение пространства информационными объектами в виде конечного множества конкретных функций, связывающих (сопоставляющих) значения координатного пространства.

Исходя из описанных условий, сформированное ядро управления позволяет определить объём необходимых ресурсов для выполнения производственных заданий, а также моменты времени использования ресурсов. Для пополняемых ресурсов (материалов, инструментов и т. д.) 
такая программа однозначно определяет формирование требований (графиков) приобретения этих ресурсов в нужных объёмах и в необходимые сроки.

Система управления предприятием, построенная на основе предлагаемого ядра управления, сформированного в едином информационном пространстве, в любой момент позволяет получить информацию о состоянии объекта управления. Составленные на основе единой согласованной информации планы подразделений имеют непротиворечивый характер и нацелены на претворение единой стратегии предприятия.

Предложенная формализация построения ядра системы управления позволяет решать существующие проблемы интеграции информационных систем на платформе единого информационного пространства.

\section{Заключение}

Рассмотренные подходы реализованы при создании интегрированной системы автоматизированного управления ресурсами для предприятия АО «Дальрыбтехцентр», выпускающего оборудование для рыбоперерабатывающих судов. Автоматизированная система конструкторскотехнологической и планово-производственной подготовки разработана на отечественной платформе «1С: УПП». В результате внедрения этой системы на предприятии основательно реконструирована организационная структура, обеспечивающая чёткую схему «прохождения» заказов по службам.

Практика внедрения и эксплуатации рассматриваемой системы показала, что наиболее ощутимый эффект наглядно проявился через полгода, когда накопилась некоторая «критическая масса» информации как конструкторско-технологической, так и плановой. С использованием автоматизированной системы процедура планирования стала выполняться в десятки раз быстрее. Например, для формирования приблизительного плана с перечнем необходимых материалов для среднестатистического изделия, даже периодически выпускаемого, опытный специалист по подготовке производства тратил три рабочих дня (24 часа), а с помощью системы эта процедура занимает около одного часа.

Общий эффект от внедрения выразился в сокращении затрат на материалы на 15-25 \%, снижении трудоёмкости в 2-2,5 раза при одновременном сокращении количества основных рабочих и повышении качества выпускаемой продукции.

\section{ЛИТЕРАТУРА}

1. Лелюхин, В. Е. Интегрированная система подготовки производства судоремонтного предприятия В. Е. Лелюхин, О. В. Колесникова // Судостроение. - 2015. - № 2 - с. 52-54.

2. Лелюхин, В. Е. Алгоритм планирования дискретного машиностроительного производства «Опадающие листья» / В. Е. Лелюхин, О. В. Колесникова // Автоматизация. Современные технологии. - 2016. - № 1. C. $15-19$.

3. Лелюхин, В. Е. Метод формирования плана многономенклатурного мелкосерийного и единичного машиностроительного производства / В. Е. Лелюхин, О. В. Колесникова // Учёные записки Комсомольскогона-Амуре государственного технического университета. Науки о природе и технике. - 2016. - № I-1(25). C. $57-65$.

4. Лелюхин, В. Е. Разработка модели управления предприятием на основе функционального подхода

В. Е. Лелюхин, О. В. Колесникова // Фундаментальные исследования. - 2017. - № 9-2. - с. 337-341;

5. Кирсанов, М. Н. Графы в Марlе. Задачи, алгоритмы, программы / М. Н. Кирсанов. - М.: ФИЗМАТЛИТ, 2007. $-168 \mathrm{c}$.

6. Редькин, Н. П. Дискретная математика: курс лекций для студентов-механиков / Н. П. Редькин. - СПб: Издательство «Лань», 2003. - 96 с.

7. Математическая энциклопедия. В 5 т. Т. 1. / под ред. И. М. Виноградова. - М.: Советская энциклопедия, 1977. $-576 \mathrm{c}$.

8. Spicknall, M. H. Dimensional Engineering Process for Shipbuilding / M. H. Spicknall, R. Kumar // Journal of Ship Production. - 2001. - Vol. 18. - №. 2. - P. 105-115.

9. Halevi, G. Industrial Management - Control and Profit / G. Halevi // Lecture Notes in Management and Industrial Engineering 1, Springer International Publishing Switzerland. - 2014. - 284 p.

10. Badiou, A. Being and Event / A. Badiou. - New York: Continuum, 2007. - 528 p. 